\title{
Krebsrisiko bei regelmäßiger ASS-Einnahme geringer
}

\author{
Zunehmend weisen Studien darauf hin, dass Acetylsalicylsäure (ASS) vor Dick- und Enddarm- \\ krebs schützen kann. In den USA wurde deshalb kürzlich die Einnahme von ASS zur Prävention \\ von Kolorektalkrebs für bestimmte Bevölkerungsgruppen empfohlen. Ob und unter welchen \\ Bedingungen dies auch für andere Krebsarten gilt, haben Forscher jetzt untersucht.
}

Für ihre Untersuchung nutzten die Autoren zwei große prospektive Kohortenstudien: die „Nurses“ Health Study“ und die „Health Professionals Follow-up“ Studie. 135965 Teilnehmer machten über einen Zeitraum von bis zu 32 Jahren Angaben zur Einnahme von ASS. Die StandardDosis lag bei $325 \mathrm{mg}$ ASS, bei „Baby-ASSTabletten“ handelte sich um 81 mg-Dosen. Die ASS-Einnahme galt als regelmäßig, wenn sie mindestens 2-mal pro Woche erfolgte (Standard- und Niedrig-Dosis).

Eine regelmäßige Einnahme war mit einem insgesamt geringeren Risiko für Krebserkrankungen assoziiert (Relatives Risiko 0,97; 95\%-Konfidenzintervall 0,94$0,99)$. Dies ging in erster Linie auf die geringere Inzidenz für Tumore des Magen-
Darm-Trakts zurück (0,85; [0,80-0,91]), wobei der Effekt bei Kolorektalkrebs am größten war $(0,81$; $[0,75-0,88])$. Signifikante geschlechtsspezifische Unterschiede fanden sich ebenso wenig wie eine signifikante Assoziation zwischen regelmäßiger ASS-Einnahme und dem Risiko für Brust-, Prostata- oder Lungenkrebs. Die schützende Wirkung von ASS bei gastrointestinalen Krebsarten kam zum Tragen, wenn mindestens 0,5-1,5 Standard-ASSTabletten pro Woche bzw. eine BabyASS-Tablette pro Tag eingenommen wurde. Die regelmäßige Einnahme musste über mindestens 6 Jahre erfolgen, damit es zu dem schützenden Effekt kam.

Genaue Mechanismen, die erklären könnten, warum ASS so selektiv auf Krebs- arten des Verdauungstrakts wirkt, sind noch unbekannt. Da ihre Untersuchung keinen kausalen Zusammenhang nachweist, geben die Autoren keine allgemeine Empfehlung zu ASS als Krebsvorbeugung. Bei Personen, die Blutverdünner einnehmen müssen, oder bei familiärer Darmkrebs-Vorbelastung, könne ASS jedoch empfohlen werden. Ferner könne die Einnahme eine potenzielle kostengünstige Alternative zur endoskopischen Vorsorge oder eine Ergänzung zu bereits implementierten Screening-Programmen sein.

Mirka Homrich, Bonn

DOI 10.1055/s-0042-111605

Cao et al. The Population Impact of ... JAMA Oncol 2016; 2: 762-769

\section{Kommentar aus der Praxis}

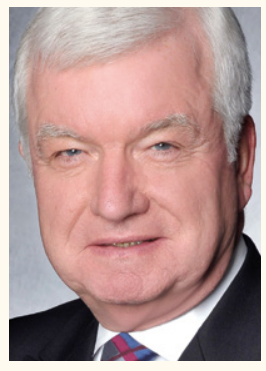

Prof. Dr. Jürgen F. Riemann

Es ist schon erstaunlich, dass die gute alte Acetylsalicylsäure (ASS), die als Schmerzmittel und Fieber senkendes Medikament schon seit über 100 Jahren erfolgreich eingesetzt wird, in den letzten Jahren eine Renaissance erfährt. Dies kommt nicht zuletzt daher, dass ASS nicht nur die Prostaglandinsynthese hemmt, sondern auch eine zunehmend beachtete antikanzerogene Wirkung besitzt. Deren zugrundeliegenden Mechanismen werden nach wie vor erforscht.

Zudem trägt die hierzulande noch unterentwickelte Versorgungsforschung einen maßgeblichen Anteil. Vor allem Kardiolo- gen und Epidemiologen haben in Langzeitstudien zunehmend beobachtet, dass die langjährige Einnahme von ASS, sei es aus kardiovaskulärer oder anderer Indikation, zu einer deutlichen Abnahme von Krebserkrankungen des Verdauungstraktes beiträgt. In einem aktuellen Review stellen Santilli et al. fest, dass ASS in der Tat einen chemopräventiven Effekt besitzt. Dieser wirkt neben der bekannten Vorbeugung von Atherothrombosen auch gegen Karzinome, besonders des Gastrointestinaltraktes.

Die amerikanische Task-Force für Präventionsleistungen (USPSTF) empfiehlt in ihrer neuesten Mitteilung niedrig dosierte ASS bei Erwachsenen von 50 bis 59 Jahren für kardiovaskuläre Erkrankungen und Darmkrebs. Grundlage hierfür sind 2 prospektive Kohortenstudien mit sehr großen Fallzahlen. Die sorgfältige Analyse unterstreicht den Effekt einer langjährigen, täglichen Einnahme von niedrig dosierter ASS besonders bei Darmkrebs.
Inzwischen ist bekannt, dass speziell die Träger von Mutationen im PI3KCA-Gen von einer ASS-Therapie nach operiertem Darmkrebs profitieren. So bekommt die Aufklärung weiterer spezifischer Biomarker besondere Bedeutung. Man kann davon ausgehen, dass sich die präventive Wirkung von ASS bei Darmkrebs noch deutlich verbessern wird, wenn sich die Einnahme auf Personen mit einem geeigneten genetischen Profil konzentriert. Die aktuelle Evidenz unterstützt jedenfalls den Effekt von ASS auf Darmkrebs und bildet den Grundstein dafür, ASS als chemopräventives Mittel einzusetzen.

Prof. Dr. Jürgen F. Riemann FACP Internist/Gastroenterologe Vorstandsvorsitzender der Stiftung LebensBlicke und em. Direktor der Med. Klinik C am Klinikum LU

Interessenkonflikt

Der Autor gibt an, dass kein Interessenkonflikt besteht. 